\title{
Evaluation of a Core of the U.S. Capsicum Germplasm Collection for Reaction to the Northern Root-knot Nematode
}

\author{
Judy A. Thies ${ }^{1}$ and Richard L. Fery ${ }^{2}$ \\ U.S. Vegetable Laboratory, Agricultural Research Service, U.S. Department \\ of Agriculture, 2875 Savannah Highway, Charleston, SC 29414-5334
}

\begin{abstract}
Additional index words. pepper, Capsicum annuum, Capsicum chinense, Capsicum baccatum, Capsicum frutescens, nematode resistance, Meloidogyne hapla
\end{abstract}

\begin{abstract}
Several species of root-knot nematodes [Meloidogyne incognita (Kofoid \& White) Chitwood, M. arenaria (Neal) Chitwood, M. javanica (Treub) Chitwood, and M. hapla Chitwood] are major pests of peppers (Capsicum spp.) in the United States and worldwide. Resistance to $M$. incognita, $M$. arenaria, and $M$. javanica has been identified in several Capsicum accessions, but there are few reports of resistance to $M$. hapla. Therefore, we selected a $10 \%$ core (440 accessions) of the 14 available Capsicum spp. in the Capsicum germplasm collection (3,731 accessions) maintained by the U.S. Dept. of Agriculture (USDA), and evaluated this core for resistance to $M$. hapla in unreplicated greenhouse tests. The 11 best (most resistant) and the 3 worst (most susceptible) accessions identified in these unreplicated tests were re-evaluated in a replicated greenhouse test. Seven of these 11 "best" accessions (PI 357613, PI 357503, PI 439381, PI 297493, PI 430490, PI 267729, and PI 441676) exhibited root gall severity indices $<5.0(1=$ no galls; $9=$ more than $80 \%$ of the root system covered with galls) in the replicated test, and each of these indices was significantly lower than the indices of the "worst" accessions and susceptible controls. Although a gall index $<5.0$ indicates a moderate level of resistance, more than $3000 \mathrm{M}$. hapla eggs were extracted per gram of fresh root tissue and the reproductive index was $>1.0$ for each of these accessions. These observations suggest that the most resistant accessions tested are somewhat susceptible to $M$. hapla. The results of our evaluation of a core of the USDA Capsicum germplasm collection demonstrates clearly that there is significant genetic variability within the overall collection for $M$. hapla resistance. Additionally, these results identify portions of the collection where future evaluations for $M$. hapla resistance should be focused. For example, the origin of the two most promising $C$. annuum accessions (PI 357613 and PI 357503) in the core was Yugoslavia. Thus, additional accessions from this temperate region of the world should receive priority attention in any effort to identify better sources of resistance in $C$. annuum to M. hapla.
\end{abstract}

Root-knot nematodes (Meloidogyne incognita, $M$. arenaria, $M$. javanica, and $M$. hapla) are major pests of both bell and hot peppers (Capsicum spp.) in the United States and worldwide (Di Vito et al., 1992; Thies et al., 1997; Thomas et al., 1995). Fumigation with methyl bromide is the primary control method for root-knot nematodes in bell pepper, and accounts for $12 \%$ of all methyl bromide used for pre-plant soil treatment in the United States. The pending loss of methyl bromide from the U.S. market in 2005 has focused significant interest in host resistance as an alternative management tool for root-knot nematodes.

\footnotetext{
Received for publication 27 Apr. 2001. Accepted for publication 17 Oct. 2001. We thank E.L. Corley, Jr., S.W. Miller, and R. Reeves for technical assistance in conducting the experiments. Mention of a trademark, proprietary product, or vendor does not constitute a guarantee or warranty of the product by the U.S. Dept. of Agriculture and does not imply its approval to the exclusion of other products or vendors that also may be suitable.

${ }^{1}$ Research Plant Pathologist.

${ }^{2}$ Research Geneticist.
}

Resistance to $M$. incognita, $M$. arenaria, and $M$. javanica has been identified in several Capsicum germplasm sources, including $C$. annuum L., C. chacoense L., C. chinense Jacq., and $C$. frutescens $\mathrm{L}$. (Di Vito, 1986; Di Vito et al., 1992; Di Vito and Saccardo, 1986; Fery et al., 1986, 1998; Fery and Thies, 1998; Hare, 1956; Hendy et al., 1985; Thies and Fery, 2000, 2001). However, there are only two reports of resistance to M. hapla in Capsicum germplasm. Di Vito et al. (1989) observed resistance to $M$. hapla in germplasm line "58920 " of $C$. frutescens and one line, "201", of $C$. chinense. Djian-Caporalino et al. (1999) reported resistance of five pepper lines to a population of $M$. hapla from England, but variable reactions to a population of $M$. hapla from Canada and another population of $M$. hapla from La Mole, France.

The U.S. Vegetable Laboratory pepper breeding program [U.S. Dept. of Agriculture (USDA), Agricultural Research Service (ARS), Charleston, S.C.] has developed and released several pepper cultivars and germplasm lines with resistance to root-knot nematodes. 'Carolina Cayenne' is a cayennetype pepper with exceptionally high resistance to M. incognita (Fery et al., 1986; Thies et al., 1997). Recently, Fery and co-workers developed and released 'Charleston Belle' and 'Carolina Wonder', the first two bell-type pepper cultivars with resistance to $M$. incognita (Fery et al., 1998). Both of these openpollinated, bell-type cultivars are homozygous for the $N$ gene, which confers resistance to $M$. incognita, M. arenaria races 1 and 2, and M. javanica, but not to M. hapla (Thies and Fery, 2000). Fery and Thies (1997, 1998) also recently identified high levels of resistance to $M$. incognita in three Scotch Bonnettype $(C$. chinense) cultigens obtained from heirloom collections, and subsequently released the resistant Scotch Bonnet-type germplasm lines PA-353, PA-398, and PA-426 (Fery and Thies, 1998). However, these three Scotch Bonnet-type germplasm lines are also susceptible to M. hapla (Thies and Fery, 2001).

The northern root-knot nematode, M. hapla, is a major pest of peppers (Capsicum spp.) in temperate areas of the United States and the world. Meloidogyne hapla is the most common root-knot nematode in the northern United States and eastern Canada; it also occurs in every southern state and along the Pacific Coast from California northward to British Columbia (Society of Nematologists, 1984). Although the northern root-knot nematode causes serious damage to Capsicum spp., there are no sweet or hot pepper cultivars with resistance to $M$. hapla available to pepper growers in the United States or worldwide. There is a critical need for resistant pepper cultivars because of the imminent loss of methyl bromide from the U.S. market. Additionally, other nematicides are also being lost from the market due to environmental concerns and prohibitive re-registration costs. The objectives of the study reported here are 2-fold: 1) to determine the extent of genetic variation within the USDA Capsicum germplasm collection for reaction to M. hapla; and 2) to identify the portions of the collection where future efforts should be focused when searching for high levels of $M$. hapla resistance within specific Capsicum species.

\section{Materials and Methods}

Inoculum preparation and greenhouse evaluation procedures. A North Carolina isolate of M. hapla obtained from K.R. Barker (N.C. State Univ.) was cultured on 'Rutgers' tomato (Lycopersicon esculentum Mill.) in isolated greenhouse benches. Egg inocula were extracted using $0.5 \% \mathrm{NaOCl}$ (Hussey and Barker, 1973). Seeds of each accession and control entries were planted in flats containing MetroMix $^{\text {TM }} 360$ (The Scotts Co., Marysville Ohio) planting medium. About 10 to 14 d later, the seedlings were transplanted in a $12 \times 12-\mathrm{cm}$ planting arrangement into $4.0 \times 2.0 \times 0.2-\mathrm{m}$ benches containing a steam-pasteurized mixture of 2 fine washed river sand : 1 sandy loam soil (by volume). Each plot consisted of five seedlings per accession or control entry. After the seedlings were established and growing, 
the root system of each plant was inoculated with $\approx 3000$ M. hapla eggs. The greenhouse air temperature was maintained at $28 \pm 3{ }^{\circ} \mathrm{C}$. Eight weeks (replicated evaluation of selected accessions) or 12 weeks (unreplicated evaluation of core collection) after inoculation, the roots of all plants were removed from the benches, and washed and evaluated for galling severity and egg mass production using a 1 to 9 scale $(1=0 \%, 2=1 \%$ to $3 \%, 3$ $=4 \%$ to $12 \%, 4=13 \%$ to $25 \%, 5=26 \%$ to $38 \%, 6=39 \%$ to $50 \%, 7=51 \%$ to $65 \%, 8=$ $66 \%$ to $80 \%$, and $9=81 \%$ to $100 \%$ of root system galled or covered with egg masses, respectively) (Thies et al., 1997).

Unreplicated evaluation of core collection. A core of 440 accessions was selected from the USDA Capsicum germplasm collection $(3,731$ total available accessions) and evaluated for reaction to the northern root-knot nematode in unreplicated greenhouse tests. The 440 accessions $($ Plant Introductions $=\mathrm{PI})$ represented at least $10 \%$ of each geographic origin/Capsicum taxa combination in the collection. The $440 \mathrm{PIs}$ were selected in the following manner. First, the entire USDA Capsicum collection database was sorted in ascending order by PI accession number within each taxa/geographic origin (country) group. Then, the first PI (lowest accession number) and every 10th PI thereafter was selected in each taxa/country group, so that $\approx 10 \%$ of each taxa/country group was represented. If there were $<10$ PIs per taxa/country group, then the last PI in the group was selected. Five pepper accessions ('Carolina Cayenne', 'Charleston Belle', 'California Wonder', PA426, and PA-350) and 'Rutgers' tomato were randomly included with each subset of 31 PIs as reference controls. 'Carolina Cayenne' is a cayenne pepper (C. annuum) selected from a population of 'Carolina Hot' that was segregating for many traits, including resistance to $M$.

Table 1. Gall indices and egg mass indices for Capsicum spp. plant introduction (PI) accessions and controls inoculated with $M$. hapla in a greenhouse test. ${ }^{2}$

\begin{tabular}{|c|c|c|c|c|c|c|c|c|c|c|c|}
\hline $\begin{array}{l}\text { Species/ } \\
\text { origin }\end{array}$ & $\begin{array}{l}\text { Accession } \\
\text { (P.I. No.) }\end{array}$ & $\begin{array}{c}\text { Gall } \\
\text { index }\end{array}$ & $\begin{array}{c}\text { Egg } \\
\text { mass } \\
\text { index } \\
\end{array}$ & $\begin{array}{l}\text { Species/ } \\
\text { origin }\end{array}$ & $\begin{array}{l}\text { Accession } \\
\text { (P.I. No.) }\end{array}$ & $\begin{array}{c}\text { Gall } \\
\text { index }{ }^{y}\end{array}$ & $\begin{array}{c}\text { Egg } \\
\text { mass } \\
\text { index }^{y}\end{array}$ & $\begin{array}{l}\text { Species/ } \\
\text { origin }\end{array}$ & $\begin{array}{c}\text { Accession } \\
\text { (P.I. No.) }\end{array}$ & $\begin{array}{c}\text { Gall } \\
\text { index }\end{array}$ & $\begin{array}{c}\text { Egg } \\
\text { mass } \\
\text { index }^{y}\end{array}$ \\
\hline C. апnиum & & & & Hungary & 288982 & 5.6 & 6.0 & Mexico & 438646 & 7.2 & 7.2 \\
\hline Afghanistan & 127445 & 6.8 & 7.2 & Hungary & 297470 & 7.6 & 8.0 & Mexico & 438657 & 6.4 & 6.4 \\
\hline Afghanistan & 256056 & 6.8 & 6.8 & Hungary & 355721 & 4.8 & 4.8 & Mexico & 438667 & 6.8 & 6.8 \\
\hline Argentina & 260453 & 5.2 & 5.4 & Hungary & 532983 & 7.4 & 7.6 & Mexico & 439269 & 5.6 & 5.6 \\
\hline Argentina & 439208 & 5.8 & 5.8 & Hungary & 593477 & 6.2 & 6.2 & Mexico & 439279 & 6.4 & 6.6 \\
\hline Australia & 504809 & 6.6 & 6.6 & Hungary & 593601 & 6.2 & 6.2 & Mexico & 439289 & 5.6 & 5.4 \\
\hline Belize & 438538 & 6.8 & 7.0 & India & 124078 & 5.8 & 5.6 & Mexico & 439299 & 7.0 & 7.0 \\
\hline Brazil & 439214 & 5.0 & 5.0 & India & 163186 & 6.2 & 6.4 & Mexico & 555619 & 8.0 & 8.0 \\
\hline Brazil & 593573 & 6.8 & 7.0 & India & 163196 & 7.4 & 7.6 & Mexico & 574547 & 5.4 & 5.6 \\
\hline Bulgaria & 466904 & 5.2 & 5.4 & India & 164287 & 5.6 & 5.6 & Mexico & 593490 & 7.0 & 7.0 \\
\hline Bulgaria & 495792 & 7.8 & 7.8 & India & 164471 & 8.8 & 8.8 & Mexico & 593500 & 5.0 & 5.0 \\
\hline Canada & 302987 & 7.8 & 7.8 & India & 164771 & 5.4 & 5.4 & Mexico & 593510 & 7.8 & 7.8 \\
\hline Chile & 439217 & 5.8 & 5.8 & India & 165588 & 7.0 & 7.0 & Mexico & 593520 & 9.0 & 9.0 \\
\hline China & 162607 & 7.4 & 7.6 & India & 174809 & 5.8 & 6.4 & Mexico & 593530 & 5.6 & 5.8 \\
\hline China & 432799 & 4.4 & 4.4 & India & 179868 & 8.0 & 8.0 & Mexico & 593540 & 6.2 & 6.2 \\
\hline China & 432809 & 5.6 & 5.4 & India & 182925 & 5.4 & 5.4 & Mexico & 593563 & 7.6 & 7.6 \\
\hline China & 432819 & 5.2 & 5.0 & India & 183439 & 5.0 & 5.0 & Mexico & 593578 & 8.2 & 8.2 \\
\hline China & 432829 & 6.2 & 6.2 & India & 209653 & 5.0 & 5.0 & Mexico & 594116 & 8.0 & 7.8 \\
\hline China & 432839 & 5.6 & 5.6 & India & 244668 & 5.0 & 5.0 & Mexico & 594127 & 5.0 & 5.0 \\
\hline China & 436645 & 5.0 & 5.0 & India & 271462 & 5.8 & 5.8 & Mexico & 594133 & 9.0 & 9.0 \\
\hline China & 478358 & 5.6 & 5.8 & India & 297482 & 5.8 & 6.2 & Moldova & 506437 & 6.2 & 6.2 \\
\hline China & 555600 & 4.8 & 4.8 & India & 297493 & 4.2 & 4.2 & Nepal & 286419 & 5.0 & 5.0 \\
\hline Colombia & 257055 & 6.6 & 6.6 & India & 322719 & 6.2 & 7.0 & Netherlands & 439324 & 6.2 & 6.4 \\
\hline Colombia & 439218 & 5.4 & 5.4 & India & 369997 & 5.6 & 5.6 & Nicaragua & 311126 & 8.4 & 8.4 \\
\hline Costa Rica & 439219 & 5.4 & 5.4 & India & 439247 & 6.4 & 6.4 & Nigeria & 439327 & 6.4 & 6.8 \\
\hline Costa Rica & 439229 & 7.2 & 8.0 & India & 593597 & 5.2 & 5.2 & Pakistan & 593581 & 6.4 & 6.6 \\
\hline Costa Rica & 439239 & 6.6 & 6.8 & Indonesia & 470244 & 6.2 & 6.2 & Panama & 406987 & 5.8 & 5.8 \\
\hline Cuba & 267731 & 5.0 & 5.0 & Iran & 138566 & 5.4 & 5.4 & Peru & 241680 & 5.2 & 5.2 \\
\hline Eastern Europe & 224761 & 5.0 & 5.0 & Iran & 142829 & 6.2 & 6.0 & Peru & 439329 & 7.2 & 7.2 \\
\hline El Salvador & 281341 & 5.0 & 5.0 & Iran & 148628 & 6.0 & 6.0 & Peru & 593603 & 6.4 & 6.2 \\
\hline Ethiopia & 194723 & 6.6 & 6.6 & Iran & 222975 & 8.4 & 8.4 & Philippines & 439337 & 8.2 & 8.0 \\
\hline Ethiopia & 197409 & 5.2 & 5.2 & Iran & 439250 & 6.0 & 6.0 & Portugal & 249908 & 5.8 & 5.8 \\
\hline Ethiopia & 439241 & 6.2 & 6.2 & Israel & 390974 & 7.4 & 7.8 & Puerto Rico & 302665 & 5.2 & 5.2 \\
\hline Fiji & 439242 & 6.4 & 6.4 & Italy & 273415 & 5.8 & 6.0 & Romania & 439338 & 6.4 & 6.4 \\
\hline Former Soviet & & & & Japan & 593604 & 6.6 & 6.6 & South Africa & 409141 & 7.2 & 7.6 \\
\hline Union & 263110 & 8.4 & 8.4 & Kazakhstan & 562692 & 7.6 & 7.6 & Spain & 164565 & 5.6 & 5.6 \\
\hline Former Soviet & & & & Kenya & 385961 & 5.8 & 5.6 & Spain & 223033 & 7.6 & 7.6 \\
\hline Union & 435945 & 5.4 & 5.6 & Korea, South & 508432 & 6.0 & 5.8 & Spain & 262905 & 6.8 & 6.8 \\
\hline France & 195275 & 6.4 & 6.4 & Korea, South & 508440 & 6.6 & 5.6 & Spain & 297457 & 8.6 & 8.6 \\
\hline French Guiana & 343929 & 5.0 & 5.0 & Lebanon & 181734 & 7.0 & 7.0 & Sudan & 555649 & 5.4 & 5.0 \\
\hline Germany & 264662 & 5.6 & 5.6 & Madagascar & 246331 & 7.8 & 7.8 & Syria & 181934 & 6.2 & 6.2 \\
\hline Greece & 593479 & 8.0 & 8.0 & Malaysia & 368076 & 6.4 & 6.4 & Thailand & 547069 & 7.2 & 6.4 \\
\hline Guatemala & 194568 & 4.8 & 4.8 & Malaysia & 368091 & 6.8 & 6.8 & Trinidad and & & & \\
\hline Guatemala & 200724 & 5.0 & 5.0 & Mexico & 201224 & 5.0 & 5.0 & Tobago & 439342 & 6.8 & 5.8 \\
\hline Guatemala & 224451 & 7.0 & 7.0 & Mexico & 201235 & 6.6 & 6.6 & Turkey & 166998 & 6.0 & 6.0 \\
\hline Guatemala & 267739 & 5.2 & 5.2 & Mexico & 201247 & 7.2 & 7.2 & Turkey & 167244 & 6.2 & 6.2 \\
\hline Guatemala & 439244 & 6.6 & 6.6 & Mexico & 224410 & 6.6 & 6.6 & Turkey & 169115 & 8.8 & 8.8 \\
\hline Guatemala & 594134 & 5.6 & 5.6 & Mexico & 267734 & 7.8 & 7.8 & Turkey & 169126 & 6.2 & 6.4 \\
\hline Honduras & 487622 & 6.2 & 6.6 & Mexico & 281363 & 8.2 & 8.2 & Turkey & 169136 & 5.8 & 5.6 \\
\hline Hungary & 288941 & 5.8 & 5.8 & Mexico & 281379 & 7.2 & 7.2 & Turkey & 171563 & 6.8 & 6.8 \\
\hline Hungary & 288951 & 5.8 & 5.8 & Mexico & 281390 & 6.2 & 6.2 & Turkey & 172773 & 5.0 & 5.0 \\
\hline Hungary & 288962 & 7.6 & 8.0 & Mexico & 433547 & 8.6 & 8.6 & Turkey & 173776 & 7.0 & 7.0 \\
\hline Hungary & 288972 & 5.8 & 6.0 & Mexico & 438620 & 5.6 & 5.6 & Turkey & 174121 & 5.0 & 5.0 \\
\hline
\end{tabular}


incognita; it is highly resistant to $M$. incognita (Fery et al., 1986). 'Charleston Belle' is one of only two bell peppers (C. аnnиum) with resistance to M. incognita (Fery et al., 1998). 'California Wonder' is a bell pepper that is susceptible to M. incognita (Thies et al., 1997). PA427 is a Scotch Bonnet-type $C$. chinense cultigen that is resistant to $M$. incognita and PA-350 is a habanero-type $C$. chinense cultigen that is highly susceptible to $M$. incognita (Thies and Fery, 2001). Each plant was rated for severity of root galling and egg mass production 12 weeks after inoculation with $M$. hapla. Means of all accessions were calculated using the PROC
MEANS statement of SAS for Windows (SAS Institute, Cary, N.C.). The data from the controls were analyzed using the GLM procedures of SAS for Windows, v. 6.12 and the means were separated using least significant difference (LSD) procedures.

Replicated evaluation of selected accessions. Eleven accessions that exhibited the least amount of root galling [gall index (GI) $=4.0$ to 6.0 ] in response to M. hapla (referred to hereafter as "best") and three of the accessions that exhibited the most root galling ( $\mathrm{GI}=8.0$ to 9.0 ) (referred as to as "worst") were selected from various taxa/origin groups evaluated in the unreplicated test described above. These accessions were evaluated in a 5-replicate greenhouse test, and each plant was rated for severity of root galling and egg mass production eight weeks after inoculation with $M$. hapla. The fibrous roots were clipped from the tap roots of all plants in a plot, cut into $1-$ to $2-\mathrm{cm}$ pieces, and root fresh weight recorded. Meloidogyne hapla eggs were extracted from a 10-g subsample of fibrous roots using $1.0 \%$ $\mathrm{NaOCl}$ (Hussey and Barker, 1973). Numbers of eggs were counted using a stereomicroscope. Nematode reproduction was assessed by calculating the reproduction factor $(\mathrm{R})$ in

Table 1. Continued.

\begin{tabular}{|c|c|c|c|c|c|c|c|c|c|c|c|}
\hline $\begin{array}{l}\text { Species/ } \\
\text { origin }\end{array}$ & $\begin{array}{c}\text { Accession } \\
\text { (P.I. No.) }\end{array}$ & $\begin{array}{c}\text { Gall } \\
\text { index }\end{array}$ & $\begin{array}{c}\text { Egg } \\
\text { mass } \\
\text { index }^{y}\end{array}$ & $\begin{array}{l}\text { Species/ } \\
\text { origin }\end{array}$ & $\begin{array}{c}\text { Accession } \\
\text { (P.I. No.) }\end{array}$ & $\begin{array}{c}\text { Gall } \\
\text { index }\end{array}$ & $\begin{array}{c}\text { Egg } \\
\text { mass } \\
\text { index }^{y} \\
\end{array}$ & $\begin{array}{l}\text { Species/ } \\
\text { origin }\end{array}$ & $\begin{array}{c}\text { Accession } \\
\text { (P.I. No.) }\end{array}$ & $\begin{array}{c}\text { Gall } \\
\text { index }\end{array}$ & $\begin{array}{c}\text { Egg } \\
\text { mass } \\
\text { index }^{y} \\
\end{array}$ \\
\hline Turkey & 175617 & 5.4 & 5.4 & Yugoslavia & 357493 & 5.33 & 5.33 & Guatemala & 200729 & 5.20 & 5.40 \\
\hline Turkey & 176458 & 5.5 & 5.5 & Yugoslavia & 357503 & 3.20 & 3.20 & Hungary & 594136 & 5.20 & 5.20 \\
\hline Turkey & 176468 & 5.0 & 5.0 & Yugoslavia & 357513 & 4.20 & 4.20 & India & 370005 & 6.20 & 6.20 \\
\hline Turkey & 177294 & 7.0 & 7.0 & Yugoslavia & 357523 & 4.40 & 4.40 & Jamaica & 439378 & 5.60 & 5.60 \\
\hline Turkey & 179193 & 6.6 & 6.6 & Yugoslavia & 357533 & 6.00 & 6.00 & Mexico & 439381 & 4.20 & 4.20 \\
\hline Turkey & 182155 & 5.4 & 5.4 & Yugoslavia & 357553 & 8.20 & 8.60 & Paraguay & 439382 & 5.00 & 5.00 \\
\hline Turkey & 204565 & 5.4 & 5.4 & Yugoslavia & 357563 & 6.60 & 7.00 & Peru & 439391 & 8.40 & 8.40 \\
\hline Turkey & 205171 & 5.4 & 5.4 & Yugoslavia & 357573 & 7.80 & 8.20 & Peru & 439401 & 4.80 & 4.80 \\
\hline Turkey & 206421 & 5.2 & 5.2 & Yugoslavia & 357583 & 4.60 & 4.60 & Peru & 593605 & 5.00 & 5.00 \\
\hline Turkey & 281432 & 6.0 & 5.0 & Yugoslavia & 357593 & 5.80 & 5.80 & Russian Fed. & 594138 & 5.80 & 5.80 \\
\hline Turkey & 338993 & 7.4 & 7.4 & Yugoslavia & 357603 & 8.80 & 8.80 & United States & 439407 & 8.20 & 8.20 \\
\hline Turkey & 339003 & 9.0 & 9.0 & Yugoslavia & 357613 & 3.60 & 3.60 & Uruguay & 439411 & 5.00 & 5.00 \\
\hline Turkey & 339013 & 5.8 & 5.8 & Yugoslavia & 357623 & 4.20 & 3.60 & Venezuela & 439412 & 6.20 & 5.80 \\
\hline Turkey & 339023 & 5.80 & 5.80 & Yugoslavia & 357633 & 5.60 & 5.40 & \multicolumn{4}{|c|}{ C. baccatum v. baccatum } \\
\hline Turkey & 339033 & 8.33 & 8.33 & Yugoslavia & 357643 & 5.20 & 5.20 & & 337523 & 5.80 & 5.80 \\
\hline Turkey & 339043 & 8.60 & 8.60 & Yugoslavia & 368397 & 4.80 & 4.80 & Bolivia & 260569 & 5.20 & 5.60 \\
\hline Turkey & 339053 & 8.80 & 8.80 & Yugoslavia & 368407 & 5.00 & 5.00 & Bolivia & 260581 & 8.40 & 8.40 \\
\hline Turkey & 339064 & 8.60 & 8.60 & Yugoslavia & 368418 & 7.40 & 7.40 & Bolivia & 281306 & 6.00 & 6.00 \\
\hline Turkey & 339074 & 8.20 & 8.20 & Yugoslavia & 368430 & 6.40 & $\begin{array}{l}6.40 \\
5.50\end{array}$ & Bolivia & 281308 & 6.20 & 5.20 \\
\hline Turkey & 339084 & 8.00 & 8.00 & Yugoslavia & 368440 & 5.50 & 5.50 & Brazil & 260533 & 9.00 & 9.00 \\
\hline Turkey & 339094 & 9.00 & 9.00 & Yugoslavia & 368450 & $\begin{array}{l}5.40 \\
520\end{array}$ & $\begin{array}{l}5.20 \\
520\end{array}$ & Brazil & 260593 & 5.80 & 5.80 \\
\hline Turkey & 339104 & 5.00 & 5.00 & Yugoslavia & 368460 & 5.20 & 5.20 & Brazil & 441521 & 6.00 & 6.00 \\
\hline Turkey & 339115 & 8.00 & 8.20 & Yugoslavia & 368470 & 9.00 & 9.00 & Brazil & 441531 & 5.00 & 5.20 \\
\hline Turkey & 339126 & 4.00 & 4.40 & Yugoslavia & 368480 & 9.00 & 9.00 & Brazil & 441541 & 5.00 & 5.00 \\
\hline Turkey & 339137 & 9.00 & 9.00 & Yugoslavia & 370368 & 5.00 & 5.20 & Brazil & 441551 & 5.60 & 5.60 \\
\hline Turkey & 344283 & 5.60 & 5.60 & Yugoslavia & 370378 & 6.60 & 6.60 & Brazil & 441561 & 7.20 & 7.20 \\
\hline Turkey & 344293 & 7.80 & 7.80 & Yugoslavia & 370388 & 4.40 & 4.40 & Brazil & 441571 & 5.20 & 5.20 \\
\hline Turkey & 490994 & 8.20 & 8.20 & Yugoslavia & 370398 & 4.60 & 4.60 & Brazil & 441581 & 5.40 & 5.40 \\
\hline United States & 159232 & 5.40 & 5.40 & Yugoslavia & 370408 & 5.00 & 5.20 & Brazil & 441591 & 5.60 & 5.60 \\
\hline United States & 159257 & 7.60 & 7.40 & Yugoslavia & 379116 & 6.40 & 6.60 & Brazil & 441597 & 5.00 & 5.00 \\
\hline United States & 159271 & 8.20 & 8.20 & Yugoslavia & 379126 & 5.00 & 5.00 & Brazil & 497985 & 5.40 & 5.40 \\
\hline United States & 185475 & 7.80 & 7.80 & Yugoslavia & 379136 & 5.20 & 5.20 & Chile & 281321 & 8.20 & 8.20 \\
\hline United States & 273418 & 5.60 & 5.40 & Yugoslavia & 379146 & 7.00 & 7.00 & Colombia & 257130 & 5.00 & 5.00 \\
\hline United States & 342948 & 6.60 & 6.40 & Yugoslavia & 379156 & 5.40 & 5.60 & Costa Rica & 224440 & 5.40 & 5.40 \\
\hline United States & 371874 & 7.80 & 7.60 & Yugoslavia & 379166 & 6.00 & 6.20 & Ecuador & 355814 & 5.00 & 6.00 \\
\hline United States & 439349 & 8.60 & 8.60 & Yugoslavia & 379177 & 7.80 & 8.20 & Guatemala & 267729 & 4.00 & 5.00 \\
\hline United States & 555597 & 6.00 & 5.80 & Yugoslavia & 379187 & 5.80 & 5.80 & Guyana & 199506 & 5.20 & 5.40 \\
\hline United States & 586666 & 7.40 & 7.60 & Yugoslavia & 379198 & 7.60 & 7.80 & India & 370010 & 5.00 & 6.00 \\
\hline United States & 592804 & 6.60 & 6.60 & Yugoslavia & 379208 & 7.80 & 7.80 & Kenya & 321078 & 7.00 & 7.00 \\
\hline United States & 592814 & 5.40 & 5.40 & Yugoslavia & 381321 & 8.20 & 8.20 & Mexico & 266042 & 8.00 & 8.00 \\
\hline United States & 593474 & 7.80 & 7.80 & \multicolumn{4}{|c|}{ C. annuит v. annuит } & Netherlands & 273420 & 6.60 & 6.60 \\
\hline United States & 593584 & 6.40 & 6.40 & United States & 427291 & 7.80 & 7.80 & Peru & 215699 & 7.20 & 7.40 \\
\hline United States & $\begin{array}{l}603810 \\
439357\end{array}$ & 5.40 & 5.60 & C. baccatum & & & & Peru & 241656 & 6.20 & 5.20 \\
\hline $\begin{array}{l}\text { Venezuela } \\
\text { Viroin Island }\end{array}$ & $\begin{array}{l}439357 \\
294452\end{array}$ & $\begin{array}{l}6.60 \\
6.60\end{array}$ & $\begin{array}{l}6.60 \\
7.00\end{array}$ & Argentina & 439359 & 8.20 & 8.20 & Peru & 257153 & 5.00 & 5.00 \\
\hline Virgin Island & $\begin{array}{l}294452 \\
357413\end{array}$ & $\begin{array}{l}0.00 \\
5.40\end{array}$ & 5.40 & Bolivia & 260434 & 5.40 & 5.40 & Peru & 257173 & 4.60 & 4.60 \\
\hline $\begin{array}{l}\text { Yugoslavia } \\
\text { Yugoslavia }\end{array}$ & & 3.80 & 4.00 & Brazil & 497974 & 5.00 & 5.00 & Peru & 260506 & 7.00 & 7.00 \\
\hline $\begin{array}{l}\text { Yugoslavia } \\
\text { Yugoslavia }\end{array}$ & 357433 & 4.60 & 4.60 & Bulgaria & 439370 & 7.80 & 7.80 & Peru & 281414 & 6.67 & 6.67 \\
\hline $\begin{array}{l}\text { Yugoslavia } \\
\text { Yugoslavia }\end{array}$ & 357443 & 4.40 & 4.60 & Chile & 439372 & 4.40 & 4.40 & Peru & 315025 & 5.60 & 5.60 \\
\hline $\begin{array}{l}\text { Yugoslavia } \\
\text { Yugoslavia }\end{array}$ & 357453 & 5.20 & 5.20 & Colombia & 413669 & 5.60 & 5.60 & Philippines & 188803 & 5.00 & 5.00 \\
\hline $\begin{array}{l}\text { Yugoslavia } \\
\text { Yugoslavia }\end{array}$ & 357463 & 3.00 & 3.00 & Costa Rica & 439375 & 5.60 & 5.60 & United States & 159279 & 6.40 & 6.00 \\
\hline Yugoslavia & 357473 & 6.20 & 6.20 & Ecuador & 585246 & 5.20 & 5.20 & United States & 342947 & 6.80 & 6.80 \\
\hline Yugoslavia & 357483 & 6.00 & 6.00 & Ecuador & 595905 & 4.60 & 4.60 & & & & \\
\hline
\end{tabular}

Table 1 continues on following page. 
which $\mathrm{R}=\mathrm{P}_{\mathrm{f}} / \mathrm{P}_{\mathrm{i}}$, where $\mathrm{P}_{\mathrm{i}}=$ the initial inoculum level and $\mathrm{P}_{\mathrm{f}}=$ final egg recovery (Sasser et al., 1984). Eggs/g fresh root and nematode reproductive index data were $\log _{10}(x+1)$ transformed before analysis. Data were analyzed using the GLM procedure of SAS for Windows, v. 6.12, and means were separated using Duncan's multiple range test.

\section{Results and Discussion}

Unreplicated evaluation of core collection. Only 430 of the 440 Capsicum PI accessions were successfully evaluated for reaction to $M$. hapla because seeds of 10 accessions failed to germinate. None of the 430 Capsicum spp. accessions exhibited high levels of resistance to M. hapla (Table 1). Root gall severity indices ranged from 3.0 to 9.0 and egg mass indices ranged from 3.0 to 9.0 (1.0 to $2.9=$ highly resistant, 3.0 to $4.9=$ moderately resistant). However, the few accessions with root gall severity and egg mass indices $<5.00$ had poor fibrous root systems, suggesting that these accessions were not resistant. Eleven of the best (most resistant) accessions and three of the worst (most susceptible) accessions were selected for subsequent re-evaluation in a replicated greenhouse test.

Replicated evaluation of selected accessions. The 11 best accessions exhibited intermediate to susceptible reactions to $M$. hapla and the three worst accessions were susceptible (Table 2). Root gall severity and egg mass indices for the best accessions ranged from 3.96 to 5.80 and 4.00 to 5.80 , respectively. Numbers of eggs/g fresh root ranged from 3,080 to 22,061 and reproductive indices ranged from 1.7 to 8.4 for the best accessions. Root gall severity and egg mass indices for the three worst accessions ranged from 6.25 to 8.08 and 6.21 to 8.08 , respectively. Numbers of eggs/g fresh root ranged from 20,103 to 48,487 and the reproductive indices ranged from 14.1 to 28.8. The four $C$. annuum checks (PA-136, 'California Wonder', 'Charleston Belle', and 'Carolina Cayenne'), the two $C$. chinense checks (PA-426 and PA-350), and

Table 1. Continued.

\begin{tabular}{|c|c|c|c|c|c|c|c|c|c|c|c|}
\hline $\begin{array}{l}\text { Species/ } \\
\text { origin }\end{array}$ & $\begin{array}{l}\text { Accession } \\
\text { (P.I. No.) }\end{array}$ & $\begin{array}{c}\text { Gall } \\
\text { index }\end{array}$ & $\begin{array}{c}\text { Egg } \\
\text { mass } \\
\text { index }^{y} \\
\end{array}$ & $\begin{array}{l}\text { Species/ } \\
\text { origin }\end{array}$ & $\begin{array}{l}\text { Accession } \\
\text { (P.I. No.) }\end{array}$ & $\begin{array}{c}\text { Gall } \\
\text { index }\end{array}$ & $\begin{array}{c}\text { Egg } \\
\text { mass } \\
\text { index }^{y} \\
\end{array}$ & $\begin{array}{l}\text { Species/ } \\
\text { origin }\end{array}$ & $\begin{array}{c}\text { Accession } \\
\text { (P.I. No.) }\end{array}$ & $\begin{array}{c}\text { Gall } \\
\text { index }\end{array}$ & $\begin{array}{c}\text { Egg } \\
\text { mass } \\
\text { index }{ }^{y} \\
\end{array}$ \\
\hline C. cardenasii & & & & Peru & 439437 & 9.00 & 9.00 & United Kingdom & 439523 & 6.40 & 6.00 \\
\hline Bolivia & 590507 & 9.00 & 9.00 & Peru & 439447 & 6.20 & 6.20 & United States & 593613 & 8.00 & 7.80 \\
\hline C. chacoense & & & & Peru & 439457 & 5.40 & 5.40 & Vanuatu & 188479 & 6.00 & 6.00 \\
\hline Argentina & 560944 & 9.00 & 9.00 & Peru & 439467 & 8.80 & 8.80 & Venezuela & 439525 & 8.00 & 8.00 \\
\hline United States & 555612 & 9.00 & 9.00 & Philippines & 281421 & 5.00 & 5.00 & C.pubescens & & & \\
\hline C. chinense & & & & Puerto Rico & 281424 & 6.80 & 7.00 & Ecuador & 355394 & 8.60 & 8.60 \\
\hline Argentina & 260465 & 6.20 & 6.40 & Spain & 257284 & 6.60 & 7.20 & Ecuador & 585264 & 7.00 & 7.00 \\
\hline Belize & 594139 & 5.80 & 5.80 & Suriname & 446902 & 8.00 & 8.40 & Ecuador & 585274 & 8.60 & 8.60 \\
\hline Bolivia & 260490 & 8.00 & 8.00 & $\begin{array}{l}\text { Trinidad and } \\
\text { Tobago }\end{array}$ & 430475 & & & Guatemala & 593625 & 7.00 & 7.00 \\
\hline Bolivia & 543184 & 7.80 & 7.80 & $\begin{array}{l}\text { Lobago } \\
\text { United States }\end{array}$ & $\begin{array}{l}4394 / 5 \\
439477\end{array}$ & $\begin{array}{l}5.80 \\
5.60\end{array}$ & $\begin{array}{l}5.60 \\
560\end{array}$ & Guatemala & 593635 & 9.00 & 9.00 \\
\hline Brazil & 441605 & 5.40 & 5.40 & United States & $\begin{array}{l}4594 / 1 \\
593612\end{array}$ & $\begin{array}{l}5.00 \\
7.60\end{array}$ & $\begin{array}{l}5.00 \\
7.60\end{array}$ & Guatemala & 593644 & 9.00 & 9.00 \\
\hline Brazil & 441606 & 8.40 & 8.40 & Venezuela & 439484 & 6.60 & 6.60 & Capsicum sp. & & & \\
\hline Brazil & 441618 & 5.40 & 5.40 & Virgin Island & 294453 & 8.40 & 8.40 & Argentina & 560934 & 4.80 & 4.80 \\
\hline Brazil & 441628 & 5.60 & 5.60 & $C$ & & & & Belize & 438540 & 5.60 & 5.60 \\
\hline Brazil & 441641 & 5.20 & 5.20 & $\underline{\text { c.eximium }}$ & & & & Bolivia & 387832 & 5.40 & 5.60 \\
\hline Brazil & 497983 & 6.00 & 5.60 & Bolivia & 594141 & 5.40 & 5.40 & Bolivia & 543186 & 9.00 & 9.00 \\
\hline Colombia & 257079 & 7.60 & 7.40 & C. frutescens & & & & Bolivia & 543201 & 4.60 & 4.60 \\
\hline Colombia & 257105 & 6.40 & 6.40 & Afghanistan & 255661 & 6.00 & 6.20 & Brazil & 441666 & 5.60 & 5.60 \\
\hline Colombia & 257124 & 8.20 & 8.20 & Brazil & 441646 & 5.00 & 5.00 & Brazil & 441676 & 4.00 & 4.00 \\
\hline Colombia & 439421 & 9.00 & 9.00 & Brazil & 497984 & 5.00 & 5.00 & Brazil & 441692 & 4.60 & 4.60 \\
\hline Costa Rica & 439426 & 6.20 & 6.20 & China & 419039 & 7.00 & 6.80 & Brazil & 441703 & 6.20 & 6.20 \\
\hline Cuba & 209590 & 5.40 & 5.60 & Colombia & 257071 & 7.00 & 7.20 & Brazil & 441714 & 6.60 & 6.60 \\
\hline Ecuador & 241668 & 9.00 & 9.00 & Colombia & 257121 & 6.00 & 6.00 & China & 430490 & 4.20 & 4.20 \\
\hline Ecuador & 281338 & 8.20 & 8.20 & Costa Rica & 439499 & 5.60 & 5.60 & Colombia & 257112 & 6.60 & 6.60 \\
\hline Ecuador & 360725 & 6.80 & 6.00 & Costa Rica & 487623 & 4.60 & 4.60 & Former Soviet & & & \\
\hline Ecuador & 585252 & 9.00 & 9.00 & Cuba & 439506 & 5.00 & 5.00 & Union & 555648 & 6.00 & 6.00 \\
\hline Ethiopia & 197405 & 5.60 & 5.60 & Ecuador & 585255 & 5.20 & 5.20 & Guatemala & 555634 & 4.60 & 4.60 \\
\hline Fiji & 439431 & 5.00 & 5.00 & El Salvador & 439508 & 5.20 & 5.20 & Guatemala & 555645 & 6.00 & 6.00 \\
\hline Guatemala & 195301 & 6.40 & 6.40 & Ethiopia & 281342 & 6.00 & 5.00 & Honduras & 487485 & 7.20 & 7.40 \\
\hline Guyana & 281315 & 8.40 & 8.60 & Fiji & 439509 & 5.20 & 5.20 & Indonesia & 470245 & 8.80 & 8.80 \\
\hline India & 322721 & 7.00 & 6.20 & Former Soviet & & & & Kenya & 385962 & 4.80 & 4.80 \\
\hline Jamaica & 485593 & 5.40 & 5.40 & Union & 263109 & 5.40 & 5.40 & Mexico & 555626 & 8.25 & 8.25 \\
\hline Korea, South & 439432 & 5.20 & 5.20 & Guatemala & 594961 & 5.40 & 5.40 & Peru & 257148 & 5.60 & 5.40 \\
\hline Mexico & 438629 & 5.20 & 5.00 & Guyana & 194880 & 7.00 & 7.00 & Spain & 420379 & 6.20 & 6.20 \\
\hline Mexico & 438642 & 5.00 & 5.00 & India & 370009 & 4.80 & 4.80 & Venezuela & 487457 & 8.60 & 8.60 \\
\hline N. Mariana & & & & Israel & 451762 & 4.80 & 4.80 & Controls & & & \\
\hline Islands & 281353 & 5.60 & 6.20 & Malaysia & 368085 & 5.20 & 5.00 & PA-350 & & $4.38^{w}$ & $4.48^{w}$ \\
\hline Peru & 224449 & 5.33 & 5.33 & Mexico & 593615 & 5.60 & 5.60 & PA $-426^{x}$ & & 5.81 & 5.71 \\
\hline Peru & 257156 & 9.00 & 9.00 & Peru & 439516 & 4.80 & 4.80 & California Won & $\operatorname{ler}^{v}$ & 6.31 & 6.26 \\
\hline Peru & 260466 & 6.40 & 6.40 & Philippines & 446908 & 5.00 & 5.00 & Charleston Bell & & 6.53 & 6.64 \\
\hline Peru & 260498 & 8.20 & 8.40 & Portugal & 239703 & 5.40 & 5.40 & Carolina Cayen & & 6.89 & 6.87 \\
\hline Peru & 260517 & 8.00 & 8.00 & Puerto Rico & 209109 & 8.00 & 7.00 & Rutgers tomato & & 8.81 & 8.88 \\
\hline Peru & 260557 & 8.40 & 8.40 & Solomon Islands & 439521 & 5.00 & 5.00 & $\operatorname{LSD}(0.05)$ & & 0.54 & 0.54 \\
\hline Peru & 315012 & 6.40 & 6.40 & Suriname & 439522 & 5.60 & 5.20 & & & & \\
\hline Peru & 315022 & 5.80 & 5.80 & Thailand & 321387 & 5.40 & 5.40 & & & & \\
\hline
\end{tabular}

${ }^{\mathrm{z}}$ Means of five plants.

${ }^{\mathrm{y}}$ Scale of 1 to 9 , where $1=$ no galling or visible egg masses present, $2=1 \%$ to $3 \%, 3=4 \%$ to $10 \%, 4=11 \%$ to $25 \%, 5=26 \%$ to $35 \%, 6=36 \%$ to $50 \%, 7=51 \%$ to $65 \%, 8=66 \%$ to $80 \%$, and $9=81 \%$ to $100 \%$ of root system galled or covered with egg masses, respectively.

${ }^{x}$ Capsicum chinense cultigens susceptible to $M$. hapla.

${ }^{w}$ Means of 14 replicates planted in a randomized complete-block design within each greenhouse bench $(n=70)$.

${ }^{\vee}$ Capsicum annuum cultivars susceptible to M. hapla.

"Lycopersicon esculentum cultivar susceptible to $M$. hapla. 
Table 2. Gall indices, egg mass indices, numbers of Meloidogyne hapla eggs per gram fresh root, and reproductive indices of M. hapla for 14 selected Capsicum sp. plant introduction (PI) accessions and controls inoculated with $M$. hapla, in a replicated greenhouse test. ${ }^{2}$

\begin{tabular}{|c|c|c|c|c|c|c|}
\hline Pepper entry & Taxon & Origin & Gall index $^{\mathrm{y}}$ & Egg mass index ${ }^{y}$ & Eggs/g fresh root & Reproductive index $^{x}$ \\
\hline \multicolumn{7}{|l|}{ Best $^{w}$} \\
\hline PI 357613 & C. annuum & Yugoslavia & $3.96 \mathrm{a}^{\mathrm{v}}$ & $4.00 \mathrm{a}$ & $4765 a-c^{u}$ & $2.2 \mathrm{bc}^{\mathrm{u}}$ \\
\hline PI 357503 & C. annuиm & Yugoslavia & $4.20 \mathrm{ab}$ & $4.20 \mathrm{a}$ & $6004 \mathrm{a}-\mathrm{c}$ & $2.7 \mathrm{~b}$ \\
\hline PI 439381 & C. baccatum & Mexico & $4.32 \mathrm{ab}$ & $4.24 \mathrm{a}$ & $3080 \mathrm{a}$ & $1.7 \mathrm{~b}$ \\
\hline PI 297493 & C. annuиm & India & $4.52 \mathrm{a}-\mathrm{c}$ & $4.56 \mathrm{ab}$ & $7926 \mathrm{a}-\mathrm{d}$ & $5.6 \mathrm{de}$ \\
\hline PI 430490 & Capsicum sp. & China & $4.60 \mathrm{a}-\mathrm{c}$ & $4.56 \mathrm{ab}$ & $10308 \mathrm{a}-\mathrm{d}$ & $4.0 \mathrm{~b}-\mathrm{d}$ \\
\hline PI 267729 & C. baccatum & Guatemala & $4.74 \mathrm{a}-\mathrm{c}$ & $4.66 \mathrm{ab}$ & $3557 \mathrm{ab}$ & $2.4 \mathrm{bc}$ \\
\hline PI 441676 & Capsicum sp. & Brazil & $4.79 \mathrm{a}-\mathrm{c}$ & $4.79 \mathrm{a}-\mathrm{c}$ & $7288 \mathrm{a}-\mathrm{c}$ & $5.8 \mathrm{de}$ \\
\hline PI 357423 & C. annuиm & Yugoslavia & $5.00 \mathrm{a}-\mathrm{c}$ & $5.04 \mathrm{a}-\mathrm{d}$ & $22061 \mathrm{c}-\mathrm{f}$ & 8.4 ef \\
\hline PI 441641 & C. chinense & Brazil & $5.08 \mathrm{a}-\mathrm{d}$ & $5.04 \mathrm{a}-\mathrm{d}$ & $7153 \mathrm{a}-\mathrm{d}$ & $4.1 \mathrm{c}-\mathrm{e}$ \\
\hline PI 432799 & C. annuиm & China & $5.32 \mathrm{~b}-\mathrm{e}$ & $5.24 \mathrm{a}-\mathrm{e}$ & $11056 \mathrm{~b}-\mathrm{d}$ & $6.4 \mathrm{de}$ \\
\hline PI 487623 & C. frutescens & Costa Rica & $5.80 \mathrm{a}-\mathrm{e}$ & $5.80 \mathrm{a}-\mathrm{e}$ & $8311 \mathrm{a}-\mathrm{c}$ & $2.9 \mathrm{~b}-\mathrm{d}$ \\
\hline \multicolumn{7}{|l|}{ Worst $^{\mathrm{t}}$} \\
\hline PI 263110 & C. annuиm & Former Soviet Union & $6.25 \mathrm{~d}-\mathrm{f}$ & $6.21 \mathrm{~d}-\mathrm{f}$ & $37660 \mathrm{e}-\mathrm{g}$ & $28.8 \mathrm{gh}$ \\
\hline PI 241668 & C. chinense & Ecuador & $8.08 \mathrm{~g}$ & $7.96 \mathrm{~g}$ & $20103 \mathrm{~d}-\mathrm{f}$ & $14.1 \mathrm{fg}$ \\
\hline PI 439407 & C. baccatum & United States & $8.08 \mathrm{~g}$ & $8.08 \mathrm{~g}$ & $48487 \mathrm{fg}$ & $26.9 \mathrm{gh}$ \\
\hline \multicolumn{7}{|l|}{ Controls ${ }^{s}$} \\
\hline PA-136 & C. annuиm & United States & $6.28 \mathrm{~d}-\mathrm{f}$ & $6.00 \mathrm{c}-\mathrm{f}$ & $10015 \mathrm{~b}-\mathrm{d}$ & $6.5 \mathrm{~d}-\mathrm{f}$ \\
\hline $\begin{array}{l}\text { California } \\
\text { Wonder }\end{array}$ & C. апnиит & United States & $6.52 \mathrm{ef}$ & 6.40 ef & $12908 \mathrm{~cd}$ & $7.7 \mathrm{~d}-\mathrm{f}$ \\
\hline Charleston Belle & C. annuиm & United States & $6.52 \mathrm{ef}$ & $6.40 \mathrm{ef}$ & $13166 \mathrm{~b}-\mathrm{d}$ & $8.2 \mathrm{~d}-\mathrm{f}$ \\
\hline PA-426 & C. chinense & United States & $6.60 \mathrm{f}$ & 6.40 ef & $13294 \mathrm{~cd}$ & $3.6 \mathrm{~b}-\mathrm{e}$ \\
\hline Carolina & C. annuum & United States & $6.68 \mathrm{f}$ & $6.60 \mathrm{f}$ & $18234 \mathrm{c}-\mathrm{e}$ & $8.1 \mathrm{~d}-\mathrm{f}$ \\
\hline \multicolumn{7}{|l|}{ Cayenne } \\
\hline PA-350 & C. chinense & United States & $6.88 \mathrm{f}$ & $6.84 \mathrm{f}$ & $8978 \mathrm{a}-\mathrm{c}$ & $0.7 \mathrm{a}$ \\
\hline Rutgers & L. esculentum & United States & $8.76 \mathrm{~g}$ & $8.92 \mathrm{~g}$ & $84843 \mathrm{~g}$ & $41.5 \mathrm{~h}$ \\
\hline
\end{tabular}

${ }^{\mathrm{z}}$ Means of 5 replicates of 5 plants each $(\mathrm{n}=25)$.

${ }^{y}$ Root gall severity and egg mass production rated using a scale of 1 to 9 , where $1=0,2=1 \%$ to $3 \%, 3=4 \%$ to $12 \%, 4=13 \%$ to $25 \%, 5=26 \%$ to $38 \%$, $6=39 \%$ to $50 \%, 7=51 \%$ to $65 \%, 8=66 \%$ to $80 \%$, and $9=81 \%$ to $100 \%$ of root system galled or covered with egg masses, respectively.

${ }^{x}$ Reproductive index $=\mathrm{P}_{\mathrm{f}} / \mathrm{P}_{\mathrm{i}}$, where $\mathrm{P}_{\mathrm{i}}=$ initial inoculum level and $\mathrm{P}_{\mathrm{f}}=$ final egg recovery.

${ }^{\text {w}}$ Best $\mathrm{PI}=$ intermediate reaction to $M$. hapla in a preliminary greenhouse screening test of $430 \mathrm{PI}$.

'Mean separation within columns by Duncan's multiple range test, $P \leq 0.05$.

"Data were $\log _{10}(\mathrm{x}+1)$ transformed before analysis. Untransformed data are shown.

'Worst PI = highly susceptible reaction to M. hapla in a preliminary greenhouse screening test of $430 \mathrm{PI}$.

${ }^{\mathrm{s}}$ C. annuum, $C$. chinense, and Lycopersicon esculentum entries with known reactions to M. hapla.

'Rutgers' tomato were all susceptible. Numbers of $M$. hapla eggs/g fresh root and reproductive indices for 'California Wonder', 'Charleston Belle', 'Carolina Cayenne', and PA-426 were similar to results from previous growth chamber and greenhouse tests (Thies and Fery, 2001). However, PA-350 supported much lower nematode reproduction in the present study $(8,978$ eggs/g fresh root ) than in the previous growth chamber $(118,422 \mathrm{eggs} / \mathrm{g}$ fresh root) and greenhouse tests $(51,839 \mathrm{eggs} /$ $\mathrm{g}$ fresh root) (Thies and Fery, 2001) because most of the fibrous roots of PA-350 plants in the present test were deteriorated and no longer contained egg masses. We often observe this phenomenon with PA-350 because it is highly susceptible to M. hapla and other Meloidogyne spp.

Seven of the 11 "best" accessions (PI 357613, PI 357503, PI 439381, PI 297493, PI 430490, PI 267729, and PI 441676) exhibited root gall severity indices $<5.0$, and each of these indices was significantly lower than the indices of the "worst" accessions and susceptible controls. Although a gall index $<5.0$ indicates a moderate level of resistance, $>3000$ eggs were extracted per $g$ of fresh root tissue and the reproductive index was $>1.0$ for each of these accessions. This suggests that even the most resistant Capsicum accessions evaluated in this test are somewhat susceptible to $M$. hapla.

\section{Conclusions}

The results of our evaluation of a core of the USDA Capsicum germplasm collection clearly demonstrate that there is significant genetic variability in $M$. hapla resistance within the overall collection. However, none of the accessions in this core exhibited even moderately resistant reactions to $M$. hapla when evaluated using both root galling severity and nematode reproduction criteria. It is important that efforts be continued to evaluate additional accessions in the collection for better levels of resistance. The results of the present study can be used to identify portions of the collection where future evaluation emphasis should be focused. For example, the origin of the two most promising $C$. апnиum accessions (PI 357613 and PI 357503) in the core was Yugoslavia. Thus, additional accessions from this temperate region of the world should receive priority attention in any effort to identify better sources of resistance in C. апnиит to M. hapla.

\section{Literature Cited}

Di Vito, M. 1986. Population densities of Meloidogyne incognita and growth of susceptible and resistant pepper plants. NematologiaMediterrenea 14:217-221.

Di Vito, M., V. Cianciotta, and G. Zaccheo. 1992. Yield of susceptible and resistant pepper in microplots infested with Meloidogyne incognita. Nematropica 22:1-6.
Di Vito, M. and F. Saccardo. 1986. Response of inbred lines of Capsicum to root-knot nematode (Meloidogyne spp.). In EUCARPIA VIth Meeting on Genetics and Breeding on Capsicum and Eggplant, Zaragoza, Spain, 21-24 Oct. 1986. p. $119-123$.

Di Vito, M., F. Saccardo, and N. Sasanelli. 1989. Reaction of lines of Capsicum spp. to root-knot nematodes (Meloidogyne spp.). EUCARPIA VIIth meeting on Genetics and Breeding on Capsicum and eggplant, Kragujevac, Yugoslavia, 27-30 June 1989. p. 171-175.

Djian-Caporalino, C., L. Pijarowski, A. Januel, V. Lefebvre, A. Daubèze, A. Palloix, A. Dalmasso, and P. Abad. 1999. Spectrum of resistance to root-knot nematodes and inheritance of heatstable resistance in pepper (Capsicum annuиm L.). Theoretical Appl. Genet. 99:496-502.

Fery, R.L.,P.D. Dukes, and W.L. Ogle. 1986. 'Carolina Cayenne' pepper. HortScience 21:330.

Fery, R.L., P.D. Dukes, Sr., and J.A. Thies. 1998. 'Carolina Wonder' and 'Charleston Belle': Southern root-knot nematode resistant bell peppers. HortScience 33:900-902.

Fery, R.L. and J.A. Thies. 1997. Evaluation of Capsicum chinense Jacq. cultigens for resistance to the southern root-knot nematode. HortScience 32:923-926.

Fery, R.L. and J.A. Thies. 1998. PA-353, PA-398, and PA-426: Southern root-knot nematode-resistant Capsicum chinense Jacq. germplasm lines. HortScience 33:760-761.

Hare, W.W. 1956. Comparative resistance of seven pepper varieties to five root-knot nematodes. Phytopathology 46:669-672.

Hendy, H., A. Dalmasso, and M.C. Cardin. 1985. 


\section{Breeding, Cultivars, Rootstocks, \& Germplasm Resources}

Differences in resistant Capsicum annuum attacked by different Meloidogyne species. Nematologica 31:72-78.

Hussey, R.S. and K.R. Barker. 1973. A comparison of methods of collecting inocula of Meloidogyne spp., including a new technique. Plant Dis. Rpt. 57:1025-1028.

Sasser, J.N., C.C. Carter, and K.M. Hartman. 1984. Standardization of host suitability studies and reporting of resistance to root-knot nematodes. Crop Nematode Resistance Control Project, N.C. State Univ., U.S. Agency for Intl. Dev., Raleigh, N.C.
Society of Nematologists. 1984. Meloidogyne hapla Chitwood, 1949, p. 100-102. In: Nematode Geographical Distrib. Committee of the Soc. of Nematologists (eds.). Distribution of plant-parasitic nematode species in North America. Soc. of Nematologists.

Thies, J.A., J.D. Mueller, and R.L. Fery. 1997. Effectiveness of resistance to southern rootknot nematode in 'Carolina Cayenne' pepper in greenhouse, microplot, and field tests. J. Amer. Soc. Hort. Sci. 122:200-204.

Thies, J.A. and R.L. Fery. 2000. Characterization of resistance conferred by the $N$ gene to
Meloidogyne arenaria races 1 and 2, M. hapla, and $M$. javanica in two sets of isogenic lines of Capsicum annuum. J. Amer. Soc. Hort. Sci. 125:71-75.

Thies, J.A. and R.L. Fery. 2001. Characterization of Capsicum chinense cultigens for resistance to Meloidogyne arenaria, M. hapla, and $M$. javanica. Plant Dis. 85: 267-270.

Thomas, S.H., L.W. Murray, and M. Cardenas. 1995. Relationship of preplant population densities of Meloidogyne incognita to damage in three chile pepper cultivars. Plant Dis. 79:557559. 